\title{
Threshold Phenomena in Electron-Molecule Scattering
}

\author{
Michael Allan* \\ Department of Chemistry, University of Fribourg, chemin du Musée 9, CH-1700 Fribourg, Switzerland
}

\begin{abstract}
The performance of an electron energy loss spectrometer with hemispherical energy selectors has been improved in terms of low energy capacity, resolution, response function correction at low energies, sensitivity, and extension of the angular range to $180^{\circ}$. The extended capacity permitted a more detailed observation of threshold peaks and near threshold structures in halogen halides, observation of near threshold structures in $\mathrm{CO}_{2}, \mathrm{~N}_{2} \mathrm{O}$ and $\mathrm{CS}_{2}$ and the observation of selectivity in the excitation of Fermi-coupled vibrations by the ${ }^{2} \Pi_{u}$ shape resonance in $\mathrm{CO}_{2}$.
\end{abstract}

\section{Introduction}

Electron spectrometers with cylindrical or hemispherical electrostatic analyzers have been used to measure cross sections for electron-molecule collisions for several decades [1]. Despite this long history, new discoveries have recently been made using this technique, primarily thanks to enhancement of the instrumental performance in terms of low energy capacity, sensitivity, resolution, and extension of the angular range to $180^{\circ}$. This progress has been accompanied by substantial improvements of theory. In parallel, complementary new techniques have been developed, which extended electron scattering experiments to lower energies and even better resolutions [2]. Thus information on attachment processes at extremely low energies and high resolutions has been provided by new techniques utilizing laser photoelectron sources [3]. Total cross sections at very low energies have been measured using the synchrotron photoelectron source [4].

This article will review the improvements of the hemispherical analyzer instruments and present selected recent measurements of the elastic, vibrationally inelastic, and dissociative electron attachment cross sections made with this instrument at low energies.

\section{Experiment}

The spectrometer used for the present studies has already been described [5-7] and is schematically shown in Fig. 1.

The design aspects essential for its operation are:

- Homogeneity of the potentials within the electron optics is improved by differential pumping of the monochromator and the analyzer and by using only one material (molybdenum) for all electrodes (except the magnetic angle changer, which is coated by graphite).

- All operating voltages are computer controlled, so that both the incident electron beam and the analyzer acceptance cone can be focused and pointed in the

\footnotetext{
* e-mail: Michael.Allan@unifr.ch
}

proper direction over large energy ranges both in the energy-loss and the excitation function modes of operation.

- The gas beam-to-background particle density ratio is kept high by working very close to the nozzle exit $(\sim 1.5 \mathrm{~mm})$ and by using fast diffusion pumps $(400 \mathrm{~mm}$ and $150 \mathrm{~mm}$ diameter) for evacuating the sample.

- The resolution has been improved by using a rectangular pupil defining apertures, which provide ribbon-like beam profiles between the hemispheres. Best resolution was $7 \mathrm{meV}, \quad 10-12 \mathrm{meV}$ is reached routinely for elastic scattering and vibrational excitation. The resolution is reduced by Doppler broadening at scattering angles near $0^{\circ}$ and $180^{\circ}$, however, particularly at higher energies.

- The angular range of the instrument has been extended to $180^{\circ}$ with the Magnetic Angle Changer invented by Read and Channing [8].

- A small Wien filter is incorporated in front of the detector and permits the separation of scattered electrons from negative ions produced by dissociative electron attachment. This device is necessary because electrons and anions follow the same trajectories in purely electrostatic optics.

The operation of the Magnetic Angle Changer is illustrated schematically in Fig. 2. The two crucial aspects of its operation are:

- The currents in the inner and the outer coils circulate in opposite sense, resulting in an "actively shielded solenoid", generating nearly no magnetic field outside the device, where it would interfere with the operation of the electron optics.

- As a consequence of conservation of angular momentum a beam initially directed into the center of the collision volume will automatically pass the center even when deflected, for all electron energies and all strengths of the magnetic field.

The Fribourg version of the device, shown in Fig. 3, is made of thin copper tubing and water-cooled during operation. This allows a high current density in the coils and thus a small cross-section of the conductor, resulting in a "light" device which does not reduce the local pumping speed around the nozzle and interferes minimally with the gas flow. This property is essential for the measurement of the absolute cross sections and to reduce Doppler broadening. The copper tubing is covered by thin shrinkable PTFE tubing, which is painted by graphite. This coating shields the collision region from the voltage drop along the solenoid conductor and provides a uniform potential independent of the solenoid current. The potentials on 


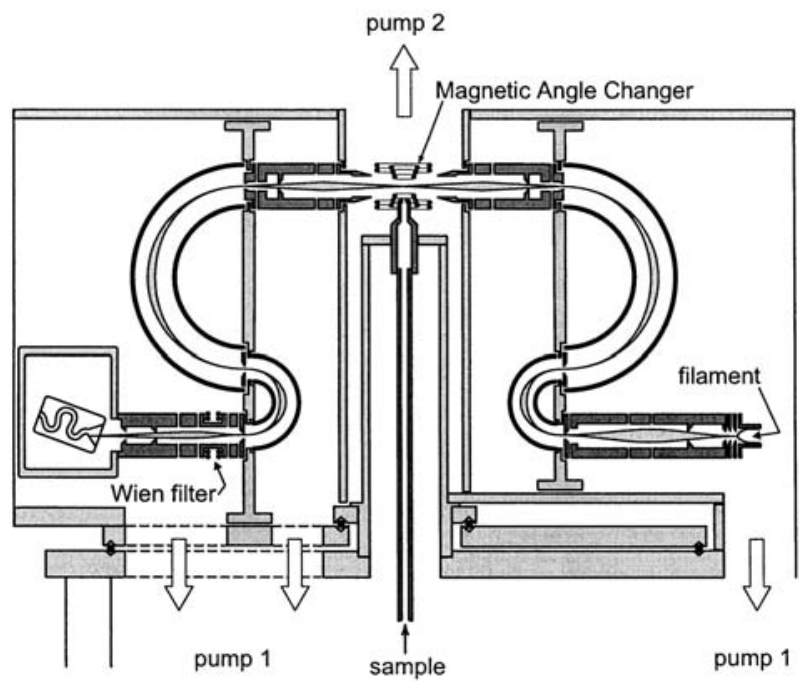

Fig. 1. Schematic diagram of the spectrometer.

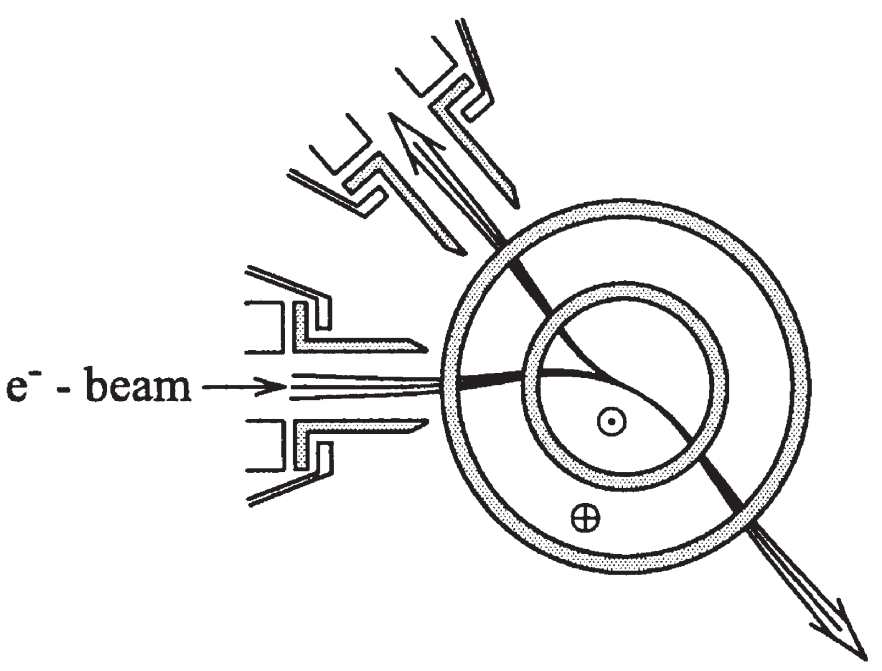

Fig. 2. Schematic diagram of the Magnetic Angle Changer. The picture shows the analyzer placed at $135^{\circ}$ and the magnetic deflection set to measure electrons elastically scattered into $180^{\circ}$.

the upper and the lower solenoids can be varied independently. An empirically determined small voltage difference (around $20 \mathrm{meV}$ ) helps to compensate the residual electric field around the nozzle and extends the low energy capacity of the instrument. The operation is simplified by the fact that the same (computer controlled) current passes both the inner and the outer solenoids.

The response function of the spectrometer for elastic scattering was determined on the elastic scattering in helium. In the present work it is assumed that the same

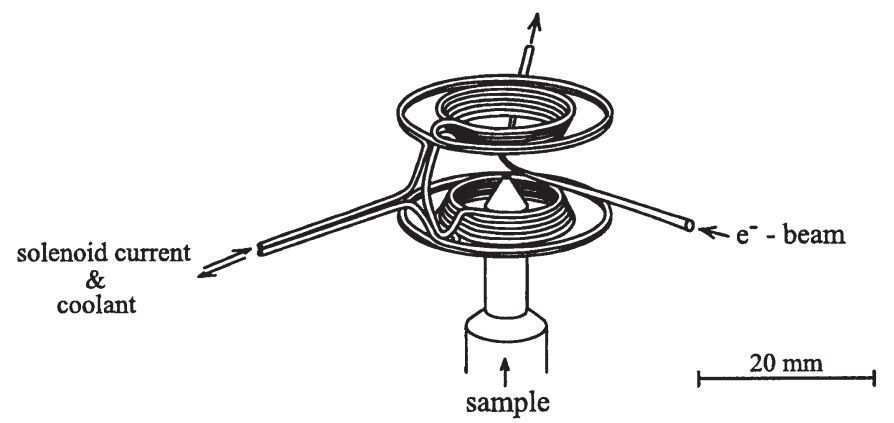

Fig. 3. Perspective drawing of the Magnetic Angle Changer used in Fribourg. response function can also be used for vibrationally inelastic scattering because the energy-losses involved are small. A comparison of inelastic and superelastic cross sections in $\mathrm{CS}_{2}$ [9] indicate that this procedure is essentially correct, but should be improved at very low energies in the future.

Absolute values of the elastic cross sections were determined by comparison with the elastic cross section of helium using the relative flow method. The gases are introduced through a single nozzle with a $0.25 \mathrm{~mm}$ diameter, kept at $\sim 30^{\circ} \mathrm{C}$ during the measurements.

The lowest energy reached by this instrument varies somewhat with time and from sample to sample, and lies between 40 and $100 \mathrm{meV}$. The performance is illustrated by the overview of the cross sections in $\mathrm{N}_{2} \mathrm{O}$ shown in Fig. 4. The present absolute values agree very well with the older measurements, indicating the degree of reliability obtained in modern absolute measurements. Similar comparison in $\mathrm{CS}_{2}$ revealed discrepancies of up to a factor of two for inelastic scattering below $1 \mathrm{eV}$ [9], however. The present measurements extend the existing data in an important way, both by providing values at lower energies, and by providing continuous excitation functions even for high vibrational overtones down to very close to threshold (within $\sim 25 \mathrm{meV}$ ). In the case of $\mathrm{N}_{2} \mathrm{O}$ the excitation functions reveal interesting sharp structures in the (200) overtone below $1 \mathrm{eV}$ which will be discussed in more detail below.

\section{Narrow structures below $\mathbf{1 e V}$}

Narrow structures below $1 \mathrm{eV}$ have initially been discovered in hydrogen halides, for example in HF where they have been interpreted as vibrational Feshbach resonances [14]. The cross sections have recently been measured with higher resolution up to $v=4$. All the features of the spectra

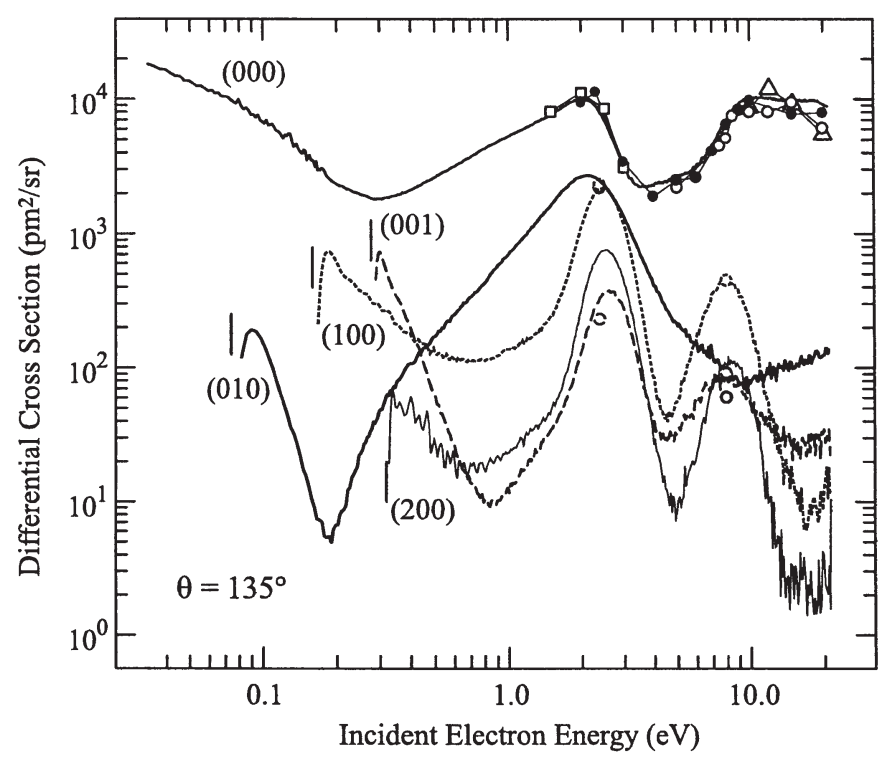

Fig. 4. Differential elastic and selected vibrational cross sections of $\mathrm{N}_{2} \mathrm{O}$ [10]. The elastic data of Marinkovic et al. [11] is shown by triangles, that of Johnstone and Newell [12] as empty circles. The elastic data of Kitajima et al. [13], extrapolated to $135^{\circ}$ from their data at $130^{\circ}$, is shown as empty squares (Sophia University data) and filled circles (Australian National University data). The vibrationally inelastic data of Kitajima et al. [13], is shown as empty circles. 


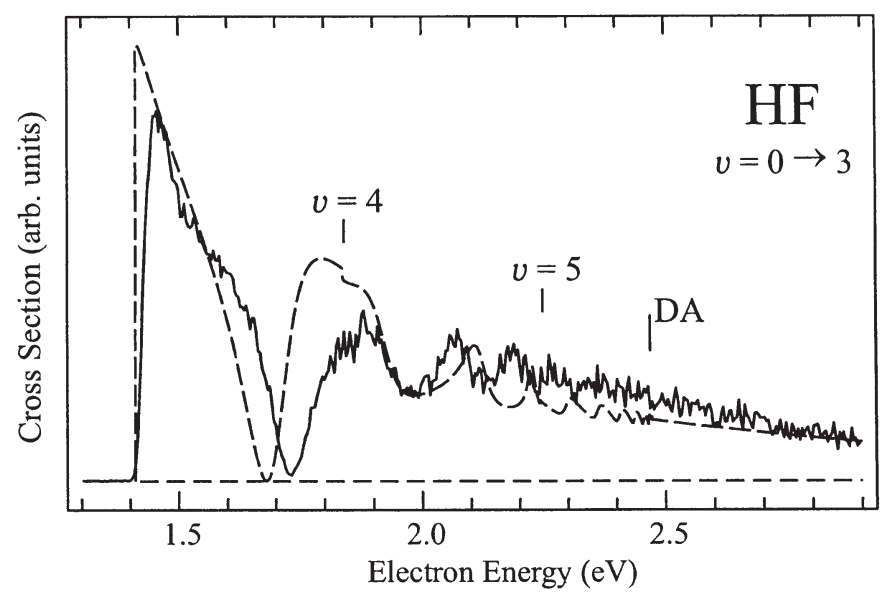

Fig. 5. Experimental and theoretical (dashed line) cross section for exciting $v=3$ in HF [15].

have been reproduced by the nonlocal resonance theory with parameters derived from ab initio calculations [15]. A sample result for $v=3$ is shown in Fig. 5. Results for other vibrational levels are shown by Hotop et al. in this issue. The qualitative features of the structures can be discussed with help of the bound part of the $\mathrm{HF}^{-}$potential curve shown in Fig. 6. At large $R$ the extra electron is bound in a valence orbital, essentially by the electron affinity of the $F$ atom. At shorter $R$ the extra electron becomes bound by the dipole moment of HF in a spatially diffuse (large) wave function. The binding becomes weaker with decreasing $R$ as the HF dipole moment diminishes until the anion and the neutral curves cross and the electron becomes unbound in the adiabatic sense. The correct description in this region is either by the nonlocal resonance theory or by the zero range potential. Despite a very large width of the resonance many oscillations of the nuclear wave packet giving rise to narrow vibrational structures - the vibrational Feshbach resonances - are possible in the anion because the electron departs only slowly and part of its cloud is re-captured

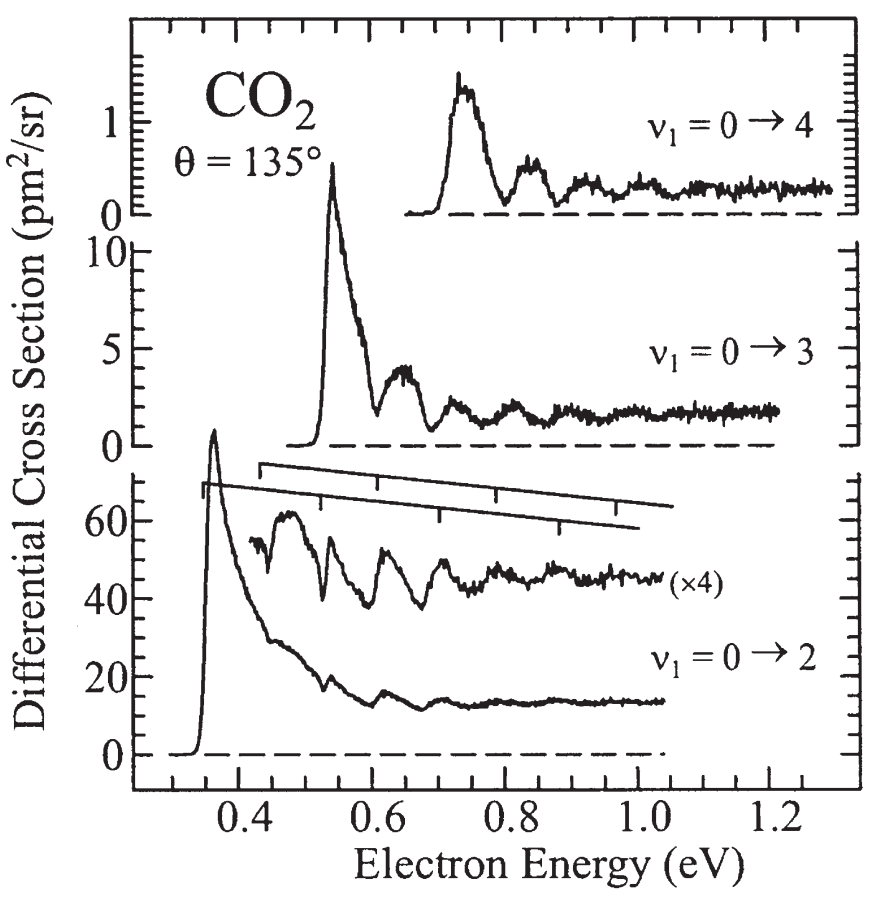

Fig. 7. Vibrational excitation cross sections in $\mathrm{CO}_{2}$. Vibrational thresholds are indicated by grids.

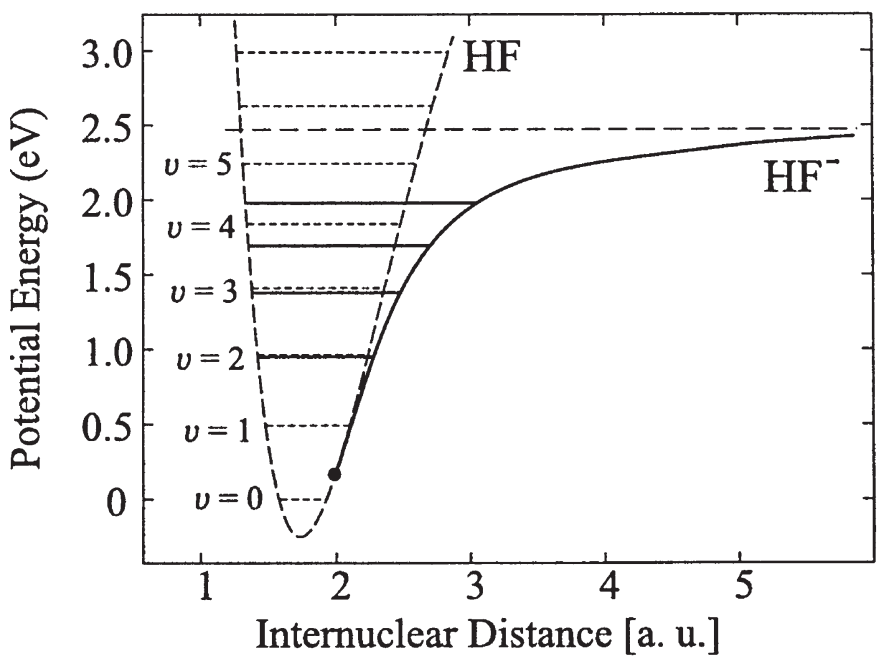

Fig. 6. Potential curves of $\mathrm{HF}$ and $\mathrm{HF}^{-}$(from reference [15]).

when the nuclear wave packet returns to larger $R$. The resonances are narrow and very close to the parent vibrational state for low $v$ 's, become broader and lie clearly below the parent level around $v=4$, and finally cause wavy 'boomerang' structure above about $1.9 \mathrm{eV}$ in the cross section in Fig. 5.

Narrow structures have recently been observed in electron scattering below $1 \mathrm{eV}$ also in $\mathrm{CO}_{2}$ [16] and the essential cross sections are shown in Fig. 7. Comparison with the HF reveals a striking phenomenological similarity of the cross sections:

- The structures are narrow at low energies and broaden at higher energies.

- The structures nearly energetically coincide with the parent vibrational levels of the neutral target at lower energies, the separation from the parent level clearly increases at intermediate energies, until they finally turn into wavy oscillations at higher energies.

- The structures deepen progressively with increasing energy and higher final states.

The phenomenological similarity suggests that the same physical principle underlies both structures as indicated by the hypothetical potential curves in Fig. 8. Whereas linear $\mathrm{CO}_{2}$ does not bind an electron (a virtual state is present), bent $\mathrm{CO}_{2}$ acquires a dipole moment which augments the binding and leads to a bound state in the fixed nuclei picture. This potential supports vibrational Feshbach resonances in a way very similar to that of $\mathrm{HF}$. The fact that the virtual state at linear geometry becomes a bound state at bent geometry has been predicted theoretically by Tennysson and Morgan [17]. The calculation of Sommerfeld has confirmed that the adiabatic potential curve of $\mathrm{CO}_{2}^{-}$bents sharply down when bent $\mathrm{CO}_{2}^{-}$is straightened [18]. Rescigno and coworkers have very recently succeeded in calculating the observed structures nearly quantitatively [19].

Narrow structures in the cross sections near threshold have also been observed in $\mathrm{N}_{2} \mathrm{O}[10]$ and $\mathrm{CS}_{2}[4,9,20]$ and representative cross sections are compared in Fig. 9. The structures in the three molecules have many similarities, but they also differ in several aspects: 


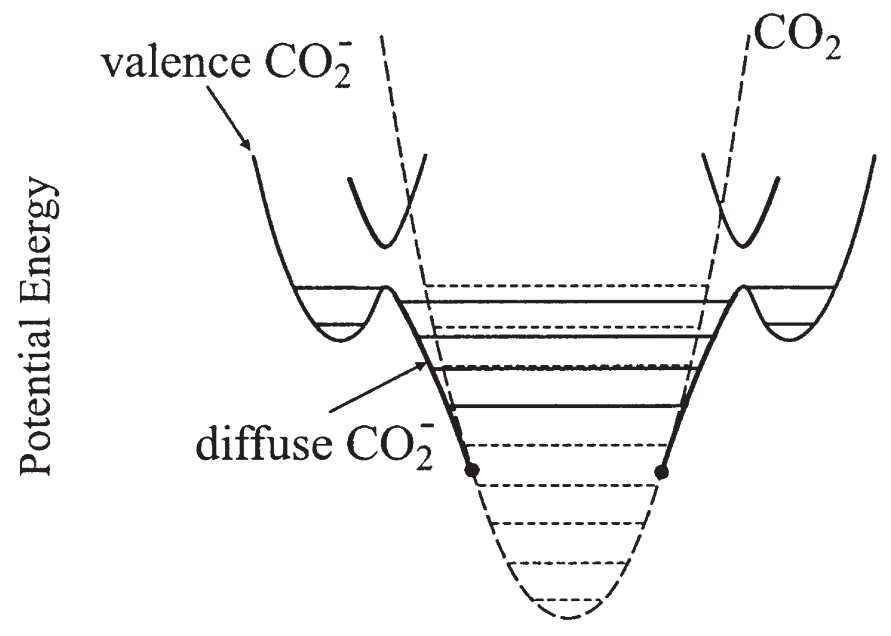

Bending and symmetric stretch

Fig. 8. Hypothetical potential curves of $\mathrm{CO}_{2}$ and $\mathrm{CO}_{2}^{-}$(from Ref. [16]).

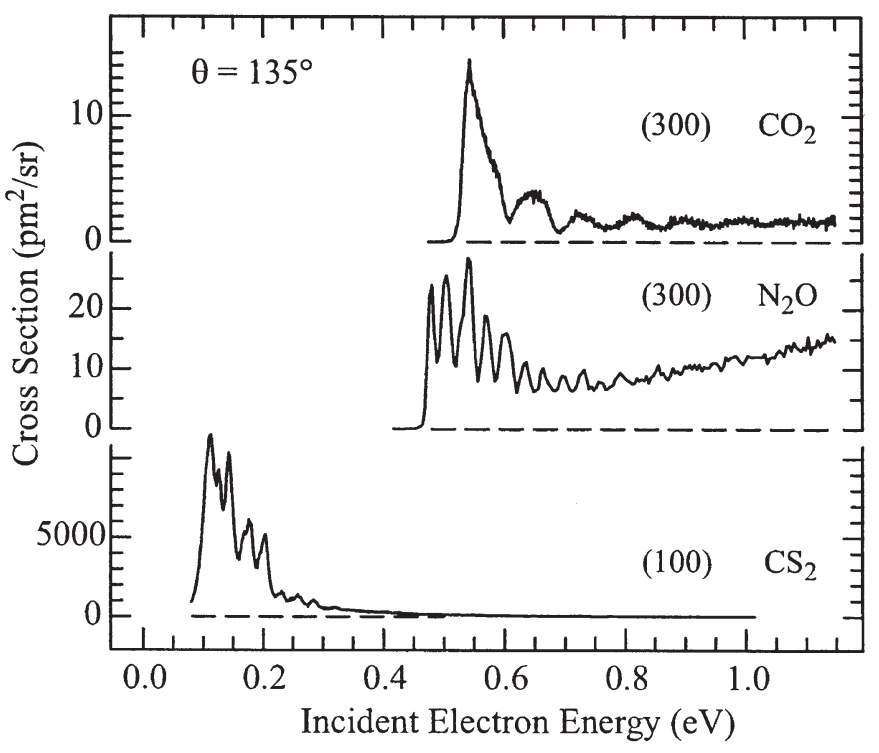

Fig. 9. Comparison of selected vibrational excitation cross sections.

- Only in $\mathrm{CS}_{2}$ does the structure appear also in the elastic cross section; the elastic cross sections in $\mathrm{N}_{2} \mathrm{O}$ and $\mathrm{CO}_{2}$ are structureless (except for a weak step at the (001) threshold in $\mathrm{CO}_{2}$ ).

- The structures in the cross sections for the excitation of the fundamental vibrations vary strongly from molecule to molecule. The cross sections for the excitation of the (010) and (100) vibrations have deep structures in $\mathrm{CS}_{2}$, only very weak structure, and only in the (100) vibration, is observed in $\mathrm{N}_{2} \mathrm{O}$, no structure is observed in the excitation of the fundamental vibrations in $\mathrm{CO}_{2}$.

- Structure is observed in all three molecules in the cross sections for the excitation of overtone vibrations involving symmetric stretch and bending, but this structure is clearly lifetime broadened in $\mathrm{CO}_{2}$, and as narrow as the instrumental resolution in $\mathrm{N}_{2} \mathrm{O}$ and $\mathrm{CS}_{2}$.

The structures in $\mathrm{CO}_{2}$ and $\mathrm{N}_{2} \mathrm{O}$ occur well below the lowest shape resonance and are assigned to vibrational Feshbach resonances supported by a "diffuse" state of the anion, where an electron is bound (in the electronic sense) by polarization and dipolar forces of the distorted target molecule. The vibrational Feshbach resonances in $\mathrm{N}_{2} \mathrm{O}$ appear also in the dissociative attachment channel, as shown in Fig. 10.

The structures in $\mathrm{CS}_{2}$ are thought to be due in majority to vibrationally excited states of the ${ }^{2} \Pi_{u}$ state of the negative ion, whose vertical electron affinity is around zero and whose ${ }^{1} A_{1}$ branch is bound. The present data does not exclude the existence of a polarizability bound diffuse state, however. Such a state may be indicated by field detachable $\mathrm{CS}_{2}^{-}$anions formed in Rydberg electron transfer [21].

\section{Excitation of the Fermi-coupled vibrations in $\mathrm{CO}_{2}$}

Striking selectivity of the excitation of the Fermi-coupled vibrations, both in the ${ }^{2} \Pi_{u}$ resonance around $3.5 \mathrm{eV}$ and the virtual state region near threshold, has been observed experimentally [7,22-25] and explained theoretically $[26,27]$.

The present work studies the angular dependence of these phenomena and the higher polyads. As an example, Fig. 11 shows the cross sections for the excitation of the three members of the triad resulting from mixing of the $\left(20^{0} 0\right),\left(12^{0} 0\right),\left(04^{0} 0\right)$ vibrations. The three members are labeled $\mathrm{FR}_{\mathrm{I}}^{\mathrm{C}}, \mathrm{FR}_{\mathrm{II}}^{\mathrm{C}}, \mathrm{FR}_{\mathrm{III}}^{\mathrm{C}}$ in the order of rising energy. Only the energetically highest member has a significant cross section at threshold - a finding which appears to be true for

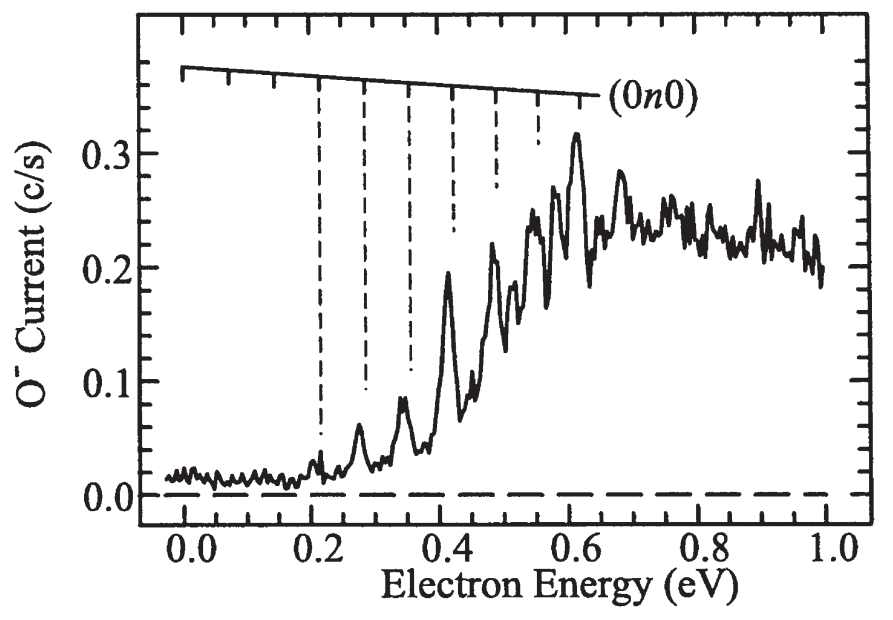

Fig. 10. Dissociative electron attachment spectrum of $\mathrm{N}_{2} \mathrm{O}$.

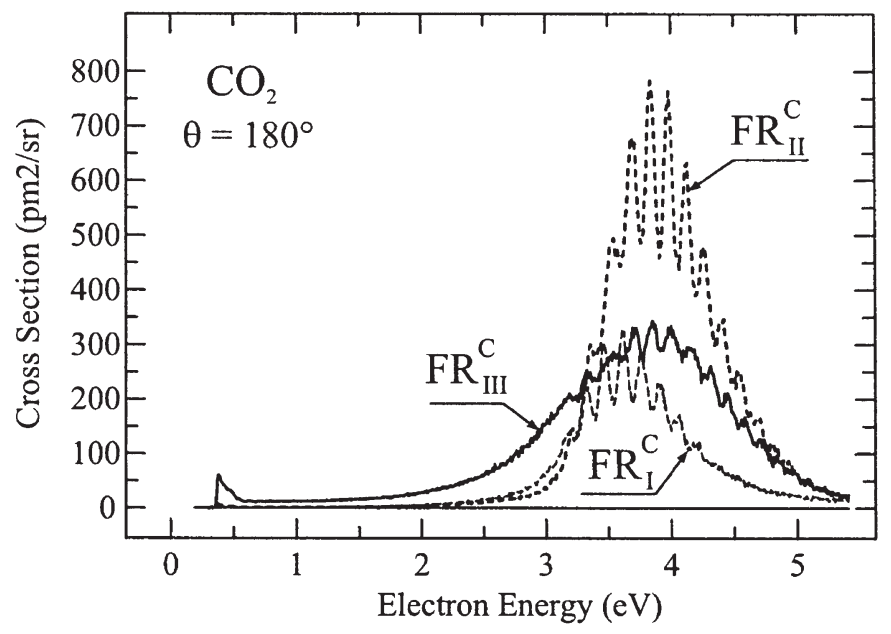

Fig. 11. Cross sections for the excitation of the Fermi triad in $\mathrm{CO}_{2}$ measured at $180^{\circ}$. 
all polyads. In the ${ }^{2} \Pi_{u}$ resonance region a pronounced dependence of the band positions and relative intensities on the scattering angle is found for each of the three polyads measured.

\section{Conclusions}

The performance of the 'classical' electron energy loss spectrometer with hemispherical energy selectors has been significantly improved over the past few years, permitting new observations, particularly in the near threshold region. The technique is complementary to the more recent techniques using photoelectron sources. Individually resolved vibrational Feshbach resonances have been observed in the halogen halides [28], methyl iodide [29,30], various clusters [2,3] and most recently also in $\mathrm{CO}_{2}$ and $\mathrm{N}_{2} \mathrm{O}$. It thus appears that vibrational Feshbach resonances associated with a "diffuse" state of the anion are not an exceptional phenomenon but occur frequently. In the case of $\mathrm{N}_{2} \mathrm{O}$ the vibrational Feshbach resonances also act as doorway states for dissociative electron attachment. The shape and peak energy of the ${ }^{2} \Pi_{u}$ band in the cross sections for the excitation of the Fermi-coupled vibrations depends strongly both on which polyad and which member of of the polyad is examined and on the scattering angle. The angular dependence is particularly pronounced around $180^{\circ}$.

\section{Acknowledgments}

This research is part of project No. 2000-067877.02 of the Swiss National Science Foundation.

\section{References}

1. Brunger, M. J. and Buckman, S. J., Phys. Rep. 357, 215 (2002).

2. Hotop, H., Ruf, M.-W., Allan, M. and Fabrikant, I. I., Adv. At. Mol. Opt. Phys., 49, 85 (2003).
3. Weber, J. M., Leber, E., Ruf, M.-W. and Hotop, H. Phys. Rev. Lett. 82, 516 (1999).

4. Jones, N. C., Field, D., Ziesel, J.-P. and Field, T. A., Phys. Rev. Lett. 89, 093201 (2002).

5. Allan, M., J. Phys. B 25, 1559 (1992).

6. Allan, M., J. Phys. B 33, L215 (2000).

7. Allan, M., Phys. Rev. Lett. 87, 033201 (2001).

8. Read, F. H. and Channing, J. M., Rev. Sci. Instrum. 67, 2372 (1996).

9. Allan, M., J. Phys. B 36, (2003) 2489.

10. Allan, M. and Skalický, T., J. Phys. B 36, 3397 (2003).

11. Marinković, B., Szmytkowski, C., Pejčev, V., Filipović, D. and Vušković, L., J. Phys. B 19, 2365 (1986).

12. Johnstone, W. M. and Newell, W. R., J. Phys. B 26, 129 (1993).

13. Kitajima, M. et al., J. Phys. B 33, 1687 (2000).

14. Knoth, G., Gote, M., Rädle, M., Jung, K. and Ehrhardt, H., Phys. Rev. Lett. 62, 1735 (1989).

15. Č́žzek, M., Horáček, J., Fabrikant, I. I. and Allan, M. J. Phys. B 36 2837 (2003).

16. Allan, M., J. Phys. B 35, L387 (2002).

17. Tennysson, J. and Morgan, L., Phil. Trans. R. Soc. Lond. A 357, 1161 (1999).

18. Sommerfeld, T., J. Phys. B 36, L127 (2003).

19. Vanroose, W., Zhang, Z., McCurdy C. W. and Rescigno, T. N., in preparation.

20. Allan, M., Abstracts of the EMS01 (2001), p. 147 and of the XXII ICPEAC (2001), p. 275

21. Suess, L., Parthasarathy, R. and Dunning, F. B., Chem. Phys. Lett. 372, 692 (2003).

22. Zhu, L., Hewitt, S. A. and Flynn, G. W., J. Chem. Phys. 94, 4088 (1990).

23. Johnstone, W. M., Akther, P. and Newell, W. R. J., Phys. B 28, 743 (1995).

24. Kitajima, M. et al., Phys. Rev. A 61, 060701 (2000).

25. Antoni, T., Jung, K., Ehrhardt, H. and Chang, E. S., J. Phys. B 19, 1377 (1986).

26. Rescigno, T. N., Isaacs, W. A., Orel, A. E., Meyer, H.-D., and McCurdy, C. W., Phys. Rev. A 65, 032716 (2002).

27. McCurdy, C. W., Isaacs, W. A., Meyer, H.-D. and Rescigno, T. N., Phys. Rev. A 67, 042708 (2003).

28. Č́žčk, M., Horáček, J., Allan, M. and Domcke, W., Czechoslovak, J., Phys. 52, 1057 (2002).

29. Schramm, A. et al., J. Phys. B 32, 2153 (1999).

30. Allan, M. and Fabrikant, I. I., J. Phys. B 35, 1025 (2002). 